\title{
Validation of Ultra High Pressure Liquid Chromatography (UHPLC) with Pre-column Derivatization Method for Quantitative Analysis of Histamine in Fish and Fishery Products
}

\author{
Karl Bryan S. Perelonia*, Flordeliza D. Cambia and Ulysses M. Montojo \\ Fisheries Post Harvest Research and Development Division - Integrated Research Laboratory \\ National Fisheries Research and Development Institute \\ Quezon City, Philippines
}

\begin{abstract}
A B S T R A C T
A pre-column derivatization-ultra high performance liquid chromatographic (UHPLC) method is described for the determination of histamine in fish and fishery products. The homogenized samples were extracted with trichloroacetic acid (TCA) solution and derivatized with o-phthaldialdehyde. Histamine was separated using reversed-phase column and determined using UHPLC with fluorescence detection. The linear calibration range was 10 to $60 \mu \mathrm{g} / \mathrm{mL}$ with a correlation coefficient of 0.9993 . Good recoveries were observed for the histamine under investigation at all spiking levels, and average recoveries were higher than $89 \%$ with a precision smaller than $8.46 \%$. The detection and quantification limit were 2.7 and $8.3 \mu \mathrm{g} / \mathrm{g}$, respectively. The uncertainty was estimated to be \pm 0.45 . The performance of the proposed method was checked with a proficiency test sample from the Food Analysis Performance Assessment Scheme (FAPAS) as external quality control; the resulting $\mathrm{z}$-score was -0.2 , which was found within acceptable range of $-2 \leq \mathrm{z} \leq 2$. The results indicated that this HPLC method was reliable, sensitive, reproducible, and practical for the routine analysis of histamine in fish and fishery products.
\end{abstract}

E-mail address: karl_perelonia8@yahoo.com

Received: January 9, 2019

Accepted: October 7, 2019
Keywords: histamine, liquid chromatography, method validation, proficiency test

\section{I N T R O D U C T I O N}

A ccording to FAO's Food Safety and Quality, Scombrotoxin Fish Poisoning (SFP), often called "histamine poisoning," is caused by ingestion of certain marine fish species containing high levels of histamine and possibly other biogenic amines (FAO 2013). These biogenic amines occur naturally in food particularly in fish by microbial decarboxylation of amino acid when fish are subjected to temperature abuse during or after harvest. Biogenic amines like histamine, putrescine, cadaverine, tyramine, and agmatine are formed from histidine, ornithine, lysine, tyrosine, and arginine, respectively. The bacteria that are considered to be involved in biogenic amines (i.e. Enterococcus; Staphylococcus; Pseudomonas; Aeromonas; Campylobacter and other Lactic acid bacteria) are present in the aquatic environment of the fish and naturally present in the normal microflora of live flesh.
Due to death, the defense mechanism of fish is no longer capable of inhibiting bacterial growth; hence histamine forming bacteria may grow in the muscle tissue (Olajos 2015).

Several methods to analyze biogenic amines in food have been described so far, including colorimetric, fluorometric, enzyme-linked immunosorbent assay (ELISA), and chromatographic methods (Peng et al. 2008). Among these methods, chromatographic technique is one of the most frequently used techniques. Although gas chromatography (GC) with flame ionization detection (FID), electron capture detection (ECD), or mass spectrometry detection (MSD) were employed for the analysis of histamine, these methods suffered from complex matrix effects and sample carryover (Jana et al. 2002). On the contrary, HPLC coupled with different detectors provided better sensitivity, selectivity, and reliability for the complex sample analysis (i.e. fish matrix). HPLC with 
ultraviolet detection has low sensitivity for histamine detection. Consequently, pre-, post-, and on-column derivatization methods are generally used to improve the detection sensitivity by using fluorescence detection (FLD). Among these three derivatization methods, the post- and on-column methods result in broader peaks of derivatives than the pre-column method due to mixing process of the analyte and reagent in the derivatization reaction device (Peng et al. 2008).

The method routinely used for histamine involves extraction with methanol or trichloroacetic acid, subsequent ion-exchange chromatography, and a chemical reaction with dansyl chloride, fluorescamine, or o-phthaldialdehyde (OPA) under defined conditions to measure the resulting fluorescence reaction products (Latorre-Moratalla et al. 2009). Dansyl chloride forms derivatives with limited stability and is itself sensitive to light. Moreover, the reaction is time-consuming even under heating. The reaction between fluorescamine and histamine, on the other hand, is not recommended due to the instability and complexity of the product; however, the reaction time is only few minutes. As the reaction between OPA and histamine completes within a minute in the presence of 3-Mercaptopropionic acid at room temperature, pre-column derivatization with OPA is commonly adapted for HPLC-FLD analysis of histamine. However, critical conditions have to be controlled to eliminate the influence of the relative instability of the derivatives (Papavergou et al. 2012).

For consumer protection, fish importing countries have regulations and varying limits for histamine in fish and fishery products. According to European Union (EC) No 2073/2005, histamine in fishery products must not exceed the following established maximum level shown in Table 1.

Indicated in the EU standard, the analytical reference method to be used for histamine analysis is HPLC. Therefore, this study was conducted to evaluate the UHPLC method for quantitative determination of histamine. The objective was to establish a rapid, sensi- tive, and reproducible analysis method and to validate it in terms of selectivity, linearity, repeatability, reproducibility, and accuracy. If the method achieved the acceptance criteria set by the Association of Official Analytical Chemist (AOAC), CODEX Alimentarius, and Food Analysis Performance Assessment (FAPAS) for all parameters of performance characteristics, then this method can be declared as fit for its intended purpose.

\section{MATERIALS AND METHODS}

\subsection{Reagents and Chemicals}

HPLC-grade acetonitrile was purchased from RCI Labscan (Bangkok, Thailand). Histamine dihydrochloride ( $\geq 99 \%)$, o-phthaldialdehyde ( $\geq 99 \%$ ), and 3-mercaptopropionic acid ( $\geq 99 \%)$ were purchased from Sigma-Aldrich (Missouri, USA). All other chemicals and solvents were analytical reagent grade and ultrapure water $(18.2 \mathrm{M} \Omega \mathrm{cm})$ produced by a Milli-Q water system (Millipore, Massachusetts, USA).

\subsection{Equipment}

The UHPLC equipment used was Shimadzu Nexera x 2 LC system (Kyoto, Japan), including a quaternary pump and fluorescence detector (excitation: $330 \mathrm{~nm}$, emission: $440 \mathrm{~nm}$ ). A personal computer equipped with a Shimadzu LabSolution program to process chromatographic data. An auto-sampler with $10 \mu \mathrm{L}$ injection valve and a $250 \times 4.6 \mathrm{~mm}$ internal diameter, $5 \mu \mathrm{m}$ particles C18 column (GL Sciences, Tokyo, Japan) thermostated to $40^{\circ} \mathrm{C}$ were used for injection and separation of analytes.

\subsection{Preparation of standard solutions and derivatization reagent}

Histamine stock solution $(1000 \mathrm{mg} / \mathrm{L})$ was prepared by dissolving $16 \mathrm{mg}$ of the compound in 10 $\mathrm{mL}$ of $100 \mathrm{mM}$ hydrochloric acid. OPA derivatization

Table 1. Histamine levels in fish and fishery products by the European Committee (EU 2073/2005)

\begin{tabular}{|lc|}
\hline \multicolumn{1}{|c|}{ Designation of products } & Regulated Limits (mg/kg wet weight) \\
\hline Raw fish & $>100 \mathrm{mg} / \mathrm{kg}$ \\
$\begin{array}{l}\text { Salted fish for species belonging to the } \\
\text { Scomboidae and Clupeidae families }\end{array}$ & $>200 \mathrm{mg} / \mathrm{kg}$ \\
$\begin{array}{l}\text { Fishery products undergoing enzyme maturation } \\
\text { treatment in brine/fish sauce }\end{array}$ & $400 \mathrm{mg} / \mathrm{kg}$ \\
\hline
\end{tabular}


reagent was prepared by dissolving $10 \mathrm{mg}$ in $10 \mathrm{~mL}$ borate buffer (100 mM, adjusted to $\mathrm{pH} 9.2$ by $1 \mathrm{M}$ sodium hydroxide). Mercaptopropionic acid solution was prepared by diluting $10 \mu \mathrm{L}$ of the compound in $10 \mathrm{~mL}$ borate buffer, $\mathrm{pH}$ 9.2. All solutions were freshly prepared.

\subsection{Sample preparation and extraction procedures}

Fresh frozen raw yellowfin tuna (Thunnus albacares) samples were carefully cut opened using a knife to remove the organs (e.g., kidney and liver). Samples were homogenized into uniform particle size before analysis using Retsch homogenizer. For extraction, $1 \mathrm{~g}$ of homogenized sample was weighed and extracted with $10 \mathrm{~mL} 0.5 \mathrm{M}$ TCA. The mixture was filtered with Whatman 40 filter paper and made up to 25 $\mathrm{mL}$ with $100 \mathrm{mM}$ borate buffer, $\mathrm{pH}$ 9.2.

\subsection{Derivatization and chromatographic separation}

Pre-column derivatization was done by adding $225 \mu \mathrm{L}$ mercaptopropionic acid and $110 \mu \mathrm{L}$ OPA solution to $37.5 \mu \mathrm{L}$ standard solution or sample extracts. The mixtures were mixed for 2 minutes prior to UHPLC determination. A linear gradient program was implemented following the conditions listed in Table 2.

\subsection{Validation design}

A full internal laboratory validation procedures were followed according to EURACHEM Guide to Method Validation, 2014. The action plan requires that the following performance characteristics must be treated and evaluated: (a) selectivity, (b) linearity, (c) precision - repeatability and reproducibility, (d) accuracy - spiking with known concentration of histamine, (e) limit of detection, (f) limit of quantification, and (g) measurement of uncertainty. Evaluation of relevant performance characteristics is shown in Table 3.

Furthermore, participation in proficiency test (canned fish, T27189) from Food Analysis Performance Assessment Scheme (FAPAS) was also conducted to further assess the reliability and accuracy of the validated analytical method.

\section{RESULTSAND DISCUSSION}

\subsection{Selectivity}

Selectivity is recommended to express whether a method can determine the target analyte (histamine) under certain chromatographic conditions in the presence of other components with similar behavior. In the chromatographic method, particularly HPLC, selectivity is based on the separation process called separation selectivity or separation factor (EURACHEM Guide for Method Validation, 2014). This selectivity indicates how strongly the result is influenced by other compounds present in the sample. The result of validation presented as chromatogram (Figure 1) shows that there is no peak detected in sample matrix as compared to the matrix spiked with $60 \mu \mathrm{g} /$ $\mathrm{mL}$ histamine, which means the method is selective for histamine and no interfering compounds eluting near histamine was observed.

Table 2. Chromatographic conditions for the sensitive analysis of histamine

\begin{tabular}{|c|c|c|}
\hline \multicolumn{3}{|c|}{ UHPLC Separation Conditions } \\
\hline LC Pump Mode & Low Pressur & ient \\
\hline Mobile Phase & $\begin{array}{l}\text { A: Acetonitr } \\
\text { B: } 0.1 \mathrm{M} \mathrm{Ace}\end{array}$ & Iffer ( $\mathrm{pH} 4.7$ at $\left.25^{\circ} \mathrm{C}\right)$ \\
\hline Detector Wavelength & $\begin{array}{l}\text { Ex: } 330 \mathrm{~nm} \\
\text { Em: } 440 \mathrm{~nm}\end{array}$ & \\
\hline Column Oven Temperature & $40^{\circ} \mathrm{C}$ & \\
\hline Flow Rate & $1.5 \mathrm{~mL} / \mathrm{min}$ & \\
\hline Injection Volume & $10 \mu \mathrm{L}$ & \\
\hline Gradient Program & $\begin{array}{l}\text { Time }(\min ) \\
0 \\
19.22 \\
47.02 \\
47.08 \\
64.66\end{array}$ & $\begin{array}{l}\text { B concentration (\%) } \\
85 \\
70 \\
60 \\
85 \\
85\end{array}$ \\
\hline
\end{tabular}


Validation of Ultra High Pressure Liquid Chromatography (UHPLC) with pre-column derivatization method for quantitative analysis of histamine in fish and fishery products

Table 3. Validation design and performance characteristics evaluated

\begin{tabular}{|c|c|c|}
\hline $\begin{array}{l}\text { Performance Characteristics to be } \\
\text { Evaluated }\end{array}$ & Procedure & $n$ (No. of Determinations) \\
\hline Linearity, Working Range & $\begin{array}{l}\text { Establish linearity by plotting the an- } \\
\text { alytical response versus the standard } \\
\text { concentration }\end{array}$ & $\begin{array}{c}\text { At least } 4 \text { standard concentrations } \\
\text { plus } 1 \text { reagent blank }\end{array}$ \\
\hline Precision (Repeatability) & $\begin{array}{l}\text { Analyze spiked sample matrix }(10 \mu \mathrm{g} / \\
\mathrm{mL}) \text { by the same analyst in the same } \\
\text { laboratory and equipment }\end{array}$ & 10 independent measurements \\
\hline $\begin{array}{l}\text { Intermediate Precision } \\
\text { (Reproducibility) }\end{array}$ & $\begin{array}{l}\text { Analyze spiked sample matrix }(10 \mu \mathrm{g} / \\
\mathrm{mL}) \text { by the same analyst in the same } \\
\text { laboratory and equipment but differ- } \\
\text { ent time scale }\end{array}$ & 10 independent measurements \\
\hline Accuracy & $\begin{array}{l}\text { Analyze blank sample matrix and } \\
\text { spike with a low, mid and high stand- } \\
\text { ard }(10,30 \text { and } 60 \mu \mathrm{g} / \mathrm{mL})\end{array}$ & 10 independent measurements \\
\hline LOD and LOQ & Analyze spiked sample matrix & 10 independent measurements \\
\hline
\end{tabular}

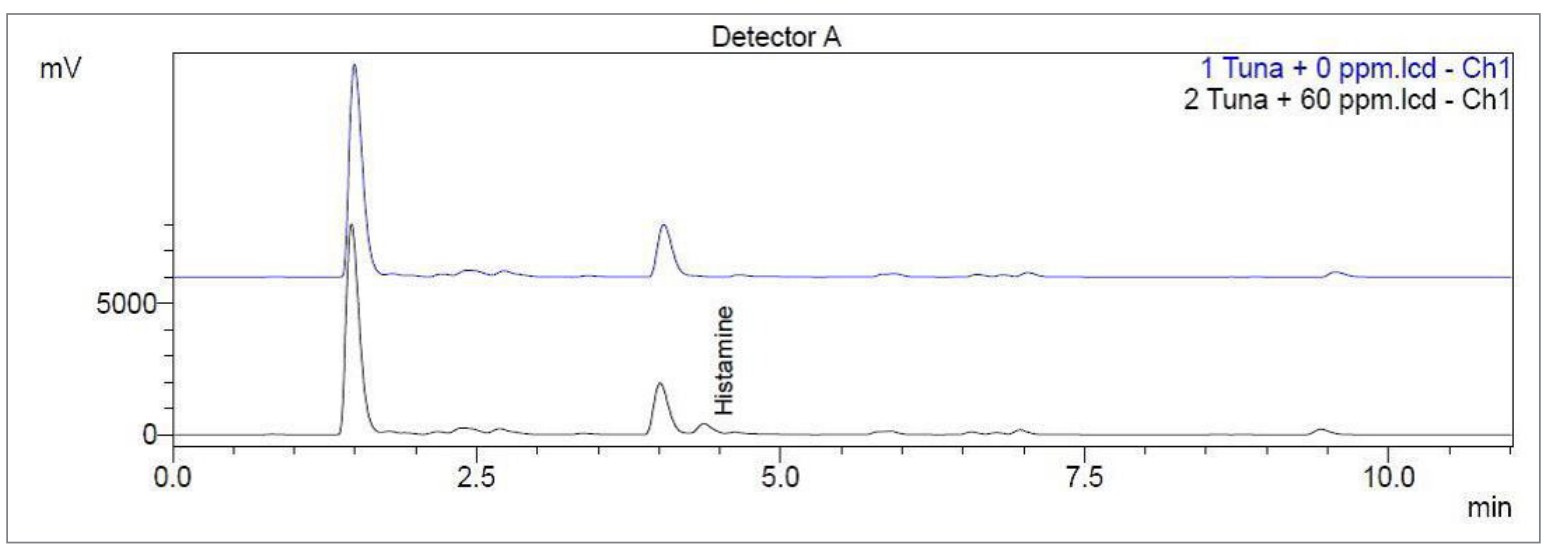

Figure 1. Chromatogram of blank sample matrix (blue) and sample matrix fortified with $60 \mu \mathrm{g} / \mathrm{mL}$ histamine (black)

\subsection{Linearity and working range}

Linearity or calibration curve was evaluated by regression analysis and least square method. The method of calibration used was standard addition to account for the selectivity. Calibration for histamine was done by standard addition method which means a matrix blank sample was spiked with 4 levels of increasing standard concentrations $(10,20,40$, and 60 $\mu \mathrm{g} / \mathrm{mL}$ ). Figure 2 shows the external calibration curve generated for two successive days. A linear curve was found between peak area and histamine concentration with good correlation coefficient $(\mathrm{R} 2=0.9999)$. The results passed the acceptance criteria based on the AOAC Peer-Verified Methods Program which is $\geq$ 0.990 .

\subsection{Precision - repeatability and reproducibility}

Precision describes the closeness between independent results under one specific chromatographic condition. This parameter is usually expressed by statistical parameters that describe the spread of results, typically the standard deviation (or relative standard deviation), calculated from results obtained by carrying out replicate measurements of spiked samples $(\mathrm{n}=$ $10)$.

Precision can be determined as repeatability and reproducibility. Repeatability is defined by EURACHEM as the variability in results when a measurement is performed by a single analyst and the same equipment on a short time scale, while intermediate precision or reproducibility measures the variability in results between two different days of analysis. 


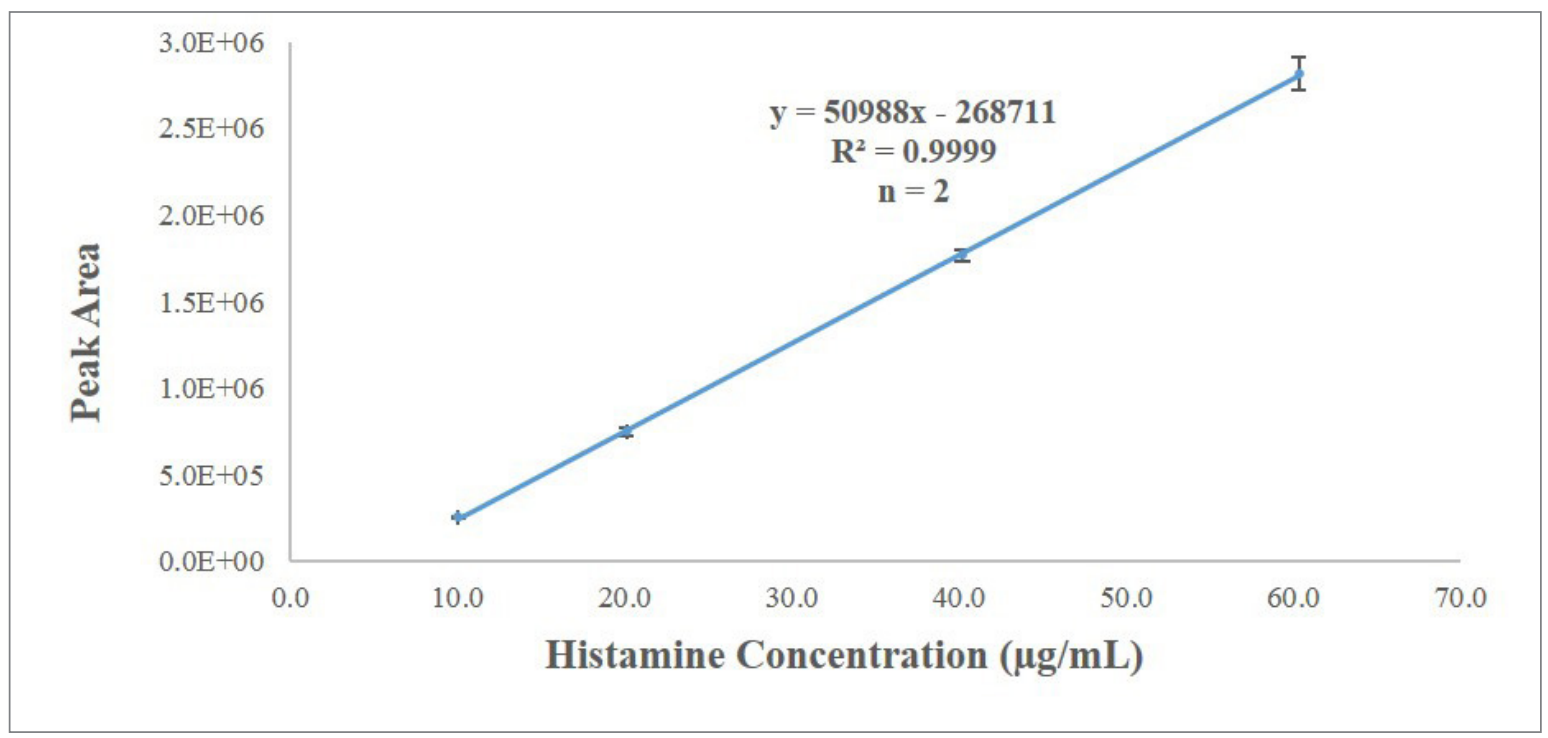

Figure 2. External standard calibration plot for histamine

Results of repeatability and intermediate precision (average and relative standard deviation for day 1 and day 2 analysis) for low-spiked samples $(10 \mu \mathrm{g} /$ $\mathrm{mL}$ ) are shown in Table 4. Furthermore, paired t-test was done to evaluate reproducibility. Results showed a significant difference $(\mathrm{p}<0.05)$ between day 1 and day 2 . This could be attributed to the preparation of standards being added in the sample. Moreover, due to thawing process, an increase in histamine levels can be observed. Nevertheless, relative standard deviation for both repeatability, for day 1 and day 2, and reproducibility passed the acceptance criterion which are $11 \%$ and $16 \%$, respectively (Table 8 ).

\subsection{Accuracy - recovery}

According to EURACHEM, measurement 'accuracy' expresses the closeness of a single result to a reference value. In this validation, accuracy in terms of recovery was evaluated by means of spiking the sample matrix with three different concentration levels of standard $(10,30$, and $60 \mu \mathrm{g} / \mathrm{mL})$. Recovery is an important step as it provides the analyst knowledge about the efficiency of the procedure. It was calculated by comparing the peak area of the fortified sample with that of the standard.

Satisfactory recoveries were observed at all spiking levels of histamine. Data from these experiments are reported in Table 5. The mean recoveries obtained are $97.52,89.68$, and $89.75 \%$ for low, mid, and high spike, respectively, which complied with the acceptance criteria of $80-110 \%$ (Table 8 ).

\subsection{Limit of detection (LOD) and limit of quantifi- cation (LOQ)}

As part of the validation study, the limit of detection (LOD) and limit of quantification (LOQ) were determined. LOD is basically defined as the lowest concentration that can be detected but not necessarily quantified. To evaluate LOD, 10 replicate of blank samples (matrix contains no detectable analyte) spiked with the lowest standard concentration, $10 \mu \mathrm{g} /$ $\mathrm{mL}$, was performed (Table 6) and LOD was calculated using equation 3.1. The LOD value was determined to be $2.4 \mu \mathrm{g} / \mathrm{g}$ histamine.

$$
\begin{aligned}
L O D= & (3 \times \text { SD of spiked sample }) \\
& (\text { Slope of the line })
\end{aligned}
$$

On the other hand, LOQ is the lowest level of analyte that can be detected and quantified with acceptable performance. 'Acceptable performance' according to EURACHEM is considered by different guidelines, which include precision and trueness. LOQ is calculated by most conventions to be the analyte concentration corresponding to the obtained LOD multiplied by a factor, $\mathrm{kQ}$. The IUPAC default value for $\mathrm{kQ}$ is 3.3. It can also be calculated using equation 3.2. The limit of quantification for this method was computed to be $8.0 \mu \mathrm{g} / \mathrm{g}$ histamine.

$$
\begin{aligned}
L O Q= & (10 \times S D \text { of spiked sample }) \\
& (\text { Slope of the line })
\end{aligned}
$$


Table 4. Precision studies data

\begin{tabular}{|ccccc|}
\hline Day & $\begin{array}{c}\text { Spiked Concentration } \\
(\mu \mathrm{g} / \mathrm{mL})\end{array}$ & $\begin{array}{c}\text { Recovered analyte } \\
\text { conc. }(\mu \mathrm{g} / \mathrm{mL})\end{array}$ & \multicolumn{2}{c|}{ RSD (\%) } \\
\cline { 3 - 5 } & & & Repeatability & Reproducibility \\
\hline 1 & 10 & $9.8000 \pm 0.8295$ & 8.46 & 6.94 \\
\hline
\end{tabular}

Table 5. Accuracy studies data

\begin{tabular}{|cccc|}
\hline $\begin{array}{c}\text { Actual concentration } \\
(\boldsymbol{\mu g} / \mathbf{m L})\end{array}$ & $\begin{array}{c}\text { Calculated concentration } \\
(\boldsymbol{\mu g} / \mathbf{m L})\end{array}$ & Recovery percentage & RSD (\%) \\
\hline 10 & $9.8000 \pm 0.8295$ & $97.52 \pm 8.25$ & 8.46 \\
30 & $27.0352 \pm 1.9432$ & $89.68 \pm 6.45$ & 10.36 \\
60 & $54.1148 \pm 5.6047$ & $89.75 \pm 9.30$ & \\
\hline
\end{tabular}

Table 6. Peak area of sample spiked with $10 \mu \mathrm{g} / \mathrm{mL}$ histamine

\begin{tabular}{|cc|}
\hline Sample & Peak Area \\
\hline Spiked Sample 1 & 238302 \\
Spiked Sample 2 & 230758 \\
Spiked Sample 3 & 237299 \\
Spiked Sample 4 & 271236 \\
Spiked Sample 5 & 317286 \\
Spiked Sample 6 & 327677 \\
Spiked Sample 7 & 297896 \\
Spiked Sample 8 & 297896 \\
Spiked Sample 9 & 216218 \\
Spiked Sample 10 & 239837 \\
\hline Average & $\mathbf{2 6 0 0 6 3}$ \\
\hline SD Spikedsample & $\mathbf{4 0 6 5 5}$ \\
\hline
\end{tabular}

\subsection{Measurement uncertainty}

The measurement of uncertainty is calculated using the guidelines for quantifying uncertainty in the analytical measurement of EURACHEM Guide. The measurand is identified as the concentration of Histamine $(\mu \mathrm{g} / \mathrm{g})$ in fish. Figure 3 shows the possible factors that can contribute to the uncertainty of measurement in the analysis, whereas Table 7 shows the summary of uncertainty sources or uncertainty budget. Calculated uncertainty for the analysis is \pm 0.45 .

\subsection{Proficiency test (PT)}

According to ISO/IEC 17043:2010, proficiency test is a method used to demonstrate compe- tency and validate a laboratory's measurement process by comparing its results to the results of a reference laboratory and other participant laboratories. Furthermore, it also enables the laboratory to validate the traceability of standards and assess the ability of laboratory personnel.

Proficiency testing schemes operate by providing participating laboratories with test samples for analysis. Laboratories analyze the test samples according to their routine procedures and report the results to the organizer. Most schemes convert the participant's result into a ' $z$-score.' This score reflects the difference between the participant's result and the accepted true value. Moreover, the consensus of participating laboratories may be calculated to determine satisfactory performance. Scores falling between -2 
and 2 indicates a satisfactory result.

A proficiency testing from the Food Analysis Performance Assessment Scheme (FAPAS) was performed to evaluate further the validated method. The assigned value for this PT round was derived from the consensus of the results submitted by the participants, and it was calculated to be $153 \mathrm{mg} / \mathrm{kg}$, as received. With this method, the laboratory generated a result of $150.8 \mathrm{mg} / \mathrm{kg}$ and has a $\mathrm{z}$-score of -0.2 , indicating that the proposed method is fit-for-purpose.

\section{C O N C L US I O N}

The reverse-phase ultra high pressure liquid chromatography method with fluorescence detection after pre-column derivatization with o-phthaldialdehyde was validated in this study. A summary of results for the different performance characteristics and their corresponding acceptance criteria is shown in Table 8. Measurement Uncertainty was calculated to be \pm 0.45 . All parameters passed the acceptance criteria set by AOAC Peer-Verified Method Program and the CODEX Alimentarius. In addition, the proposed method was confirmed through the participation of proficiency testing from FAPAS. The results $(150.8 \mathrm{mg} /$ $\mathrm{kg}$ ) agreed well with the assigned value $(153 \mathrm{mg} / \mathrm{kg})$. Overall, this UHPLC method was reliable, sensitive, reproducible, and practical for the routine analysis of histamine in fish and fishery products.

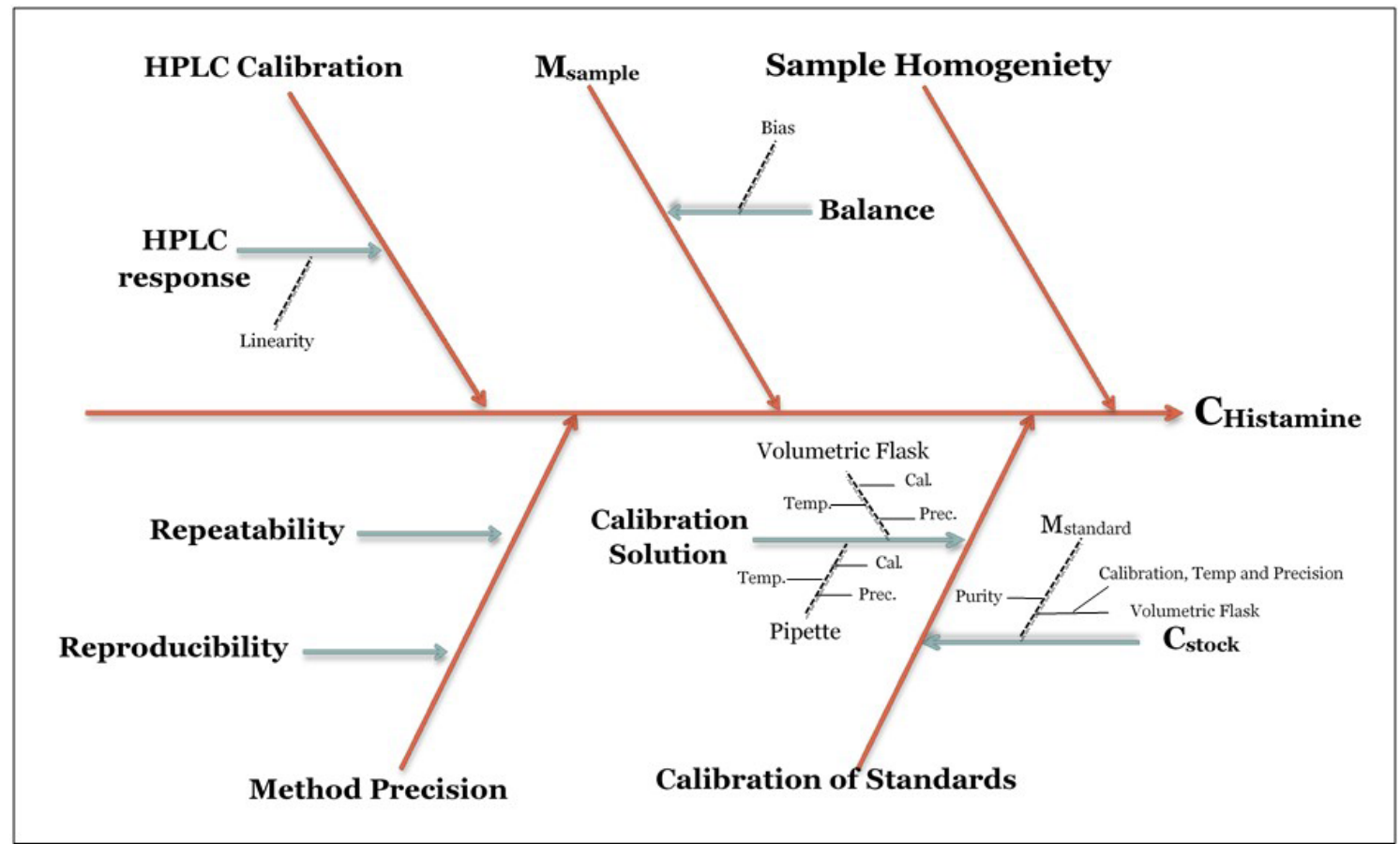

Figure 3. Cause and effect diagram for measurement uncertainty in the analysis of histamine

Table 7. Summary of uncertainty sources or uncertainty budget

\begin{tabular}{|c|c|c|c|}
\hline Source of uncertainty & Value & Standard uncertainty & $\begin{array}{l}\text { Relative standard } \\
\text { Uncertainty }\end{array}$ \\
\hline Mass sample & 5.0446 & 0.0004031 & 0.0000799 \\
\hline Mass standard & 0.1681 & 0.0004031 & 0.0023977 \\
\hline Calibration curve & 1 & 0.86822 & 0.86822 \\
\hline Volumetric Flask & 100 & 0.012250 & 0.000123 \\
\hline Volumetric Flask & 25 & 0.006124 & 0.002499 \\
\hline Pipettor & 0.1 & 0.00197 & 0.019698 \\
\hline Pipettor & 1 & 0.00347 & 0.00347 \\
\hline Method Precision & & 0.450739171 & \\
\hline
\end{tabular}




Table 8. Summary of the method parameters established in the validation
\begin{tabular}{|lll|}
\hline Performance Characteristics & Results & Acceptance Criteria \\
\hline Linearity & $\begin{array}{l}\text { Day } 1-0.9993 \\
\text { Day } 2-0.9997\end{array}$ & $\geq 0.990^{\mathrm{a}}$ \\
Precision (Repeatability) & $8.46 \%($ Day 1$)$ & $11 \% \mathrm{RSD}^{\mathrm{a}}$ \\
& $5.39 \%($ Day 2$)$ & $16 \% \mathrm{RSD}^{\mathrm{a}}$ \\
Intermediate Precision & $6.94 \%$ & \\
(Reproducibility) & $97.52 \%(10 \mu \mathrm{g} / \mathrm{mL})$ & $80-110 \%^{\mathrm{a}}$ \\
Accuracy & $89.68 \%(30 \mu \mathrm{g} / \mathrm{mL})$ & \\
& $89.75 \%(60 \mu \mathrm{g} / \mathrm{mL})$ & $<10 \mu \mathrm{g} / \mathrm{g}^{\mathrm{b}}$ \\
LOD & $2.4 \mu \mathrm{g} / \mathrm{g}$ & $<20 \mu \mathrm{g} / \mathrm{g}^{\mathrm{b}}$ \\
LOQ & $8.0 \mu \mathrm{g} / \mathrm{g}$ & \\
\hline
\end{tabular}

AOAC Peer-Verified Method

${ }^{b}$ CODEX Alimentarius

Reproducibility of this method may differ from other laboratories due to several factors such as different liquid chromatography techniques (i.e. UHPLC and HPLC), internal diameter, and length of flow lines, column dimensions, and sensitivity of the equipment. Optimization of parameters and mobile phase composition could also be done to enhance run time.

\section{A C K N O W L E G M E N T}

This study was funded by the National Fisheries Research and Development Institute as one of the component projects of the Seafood Safety and Quality Program.

The researchers would like to express their deepest gratitude to the staff of Fisheries Post Harvest Research and Development Division - Integrated Research Laboratory for the kind assistance in the collection and analyses of fish samples that were used for this study.

\section{RE F E R E N C E S}

AOAC Official Method of Analysis. 2016. Appendix F: Guidelines for Standard Method Performance Requirements. Gaitherburg, MD: AOAC International.

CODEX Alimentarius. 2017. Standard For Quick Frozen Blocks of Fish Fillet, Minced Fish Flesh and Mixture of Fillets and Minced Fish Flesh. CODEX STAN: 165-1989.

Eurachem Guide. 2014. The Fitness for Purpose of Analytical Methods (A Laboratory Guide to Method Validation and Related Topics).

FAO [Food and Agriculture Organization]. 2013. Public Health Risks of Histamine and Other Biogenic Amines from Fish and Fishery Products.
Jana L, Kathleen T, Christine W. 2002. Comparison of a capillary electrophoresis method with high performance liquid chromatography for the determination of biogenic amines in various food samples. Journal of Chromatography B 779: 229 - 239.

Latorre-Moratalla M, Lavizzari T, Vidal-Carou M. 2009. Validation of an ultra high pressure liquid chromatographic method for the determination of biologically active amines in food. Journal of Chromatography A 1216: 7715 - 7720.

Olajos I. 2015. Implementation and verification of an analytical method for the quantification of biogenic amines in seafood products. University of Iceland, School of Health Sciences.

Papavergou E, Savvaidis I, Ambrosiadis I. 2012. Levels of biogenic amines in retail market fermented meat products. Food Chemistry 135: 2750 2755 .

Peng J, Fang K, Xie D, Ding B. 2008. Development of an automated on-line pre-column derivatization procedure for sensitive determination of histamine in food with high-performance liquid chromatography-fluorescence detection. Journal of Chromatography A: 1209, 70 - 75.

Shimadzu Application News No. L449. 2013. High Speed, High Resolution Analysis of Pre-Column Derivatized Biogenic Amines by the Nexera SIL-30AC Autosampler.

Training Course Manual "Uncertainty of Measurement in Chemical Analysis". 2013. University of the Philippines - Natural Sciences Research Institute. 